\title{
The Evolution of Management
}

\section{TRANSITIONING UP THE LADDER}

\author{
KATE MATSUDAIRA
}

6. have been thinking a lot about the different transitions I have made as I have been promoted to different levels of management, from individual contributor to manager to organization leader in charge of hundreds of people.

With each step up, the job changes-but not all of the changes are obvious. You have to shift your mindset and focus on building new skills that are often very different from the skills that made you successful in your previous role.

There are lots of great resources that cover first-time managers, and many books designed for CEOs or top-level executives-but there are fewer resources specifically for the people in the middle.

Some ideas translate well from the CEOlexecutive content [such as establishing a team culture], but very little of the available content translates to running technical software teams at scale.

But this in-between space is where I have spent almost all of my careersomewhere between individual contributor (abbreviated as IC here) and CEO. Many people

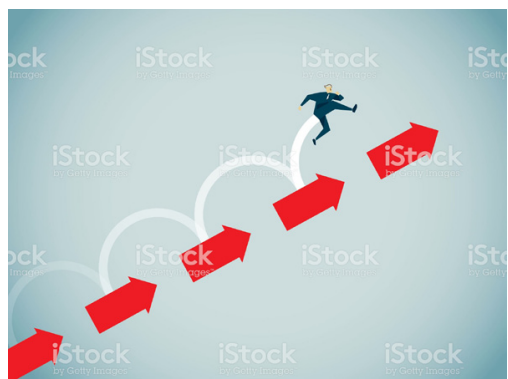


(even if they aren't yet managers) might be interested in practical advice for managing these transitions, so I have compiled everything I possibly could on the topic for this article.

INDIVIDUAL CONTRIBUTOR TO ENTRY-LEVEL MANAGER Every time you move up as a leader you go through a set of changes. One of the biggest transitions occurs when you first move from an IC role into a management position.

Your impact becomes hard to measure As an IC you are hands on and doing things yourself. You have a direct line between your daily tasks and the results: You write code for a feature for your team's product, and you can see the feature right before your eyes once you're finished. Every time your team reaches a milestone, you know exactly what you contributed to that success (and you can even quantify these contributions if you choose to].

When you move into management, you step away from that direct line. It is no longer your job to do the work yourself; instead, your role is to mentor, motivate, and guide your team to do the work, while you maintain the connection to the big-picture vision/strategy and make it easier for your team to get things done.

This can be one of the hardest parts of the job for new managers to get used to. They just want to jump in and solve problems themselves, but (as anyone who has had a manager do this knows], this actually tends to cause more harm than good. Those who try to do the work themselves can end up micromanaging or becoming a bottleneck on the project. 


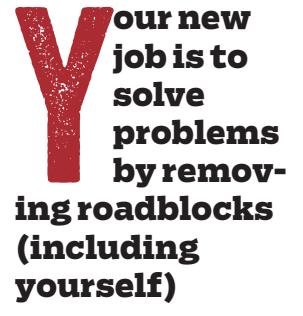

Your new job is to solve problems by removing roadblocks (including yourself), streamlining processes, and helping others be productive. You don't solve the problem yourself now; you create an environment where other people can solve the problem. This is how you add value.

This is a big shift in mindset: how you think about yourself and how you define your success.

Measurements of success become lagging indicators Unfortunately, there are not the same accolades for making that shift as there were in your previous role as an IC. Streamlining processes and mentoring your team are essential tasks but less immediately rewarding; it can sometimes take a while for the effects of your work to be truly felt and appreciated.

Understanding your impact can be elusive when the work is being done by others. And when you have a strong team, the value that you, as a leader, are bringing to the table can be hard to judge or see (this becomes even more true the higher up the ladder you go].

It is up to you to define what success means to you and your team. You achieve this by being the connection between the parent organization and your team.

\section{Communication becomes a prized skill}

Leadership is based on two-way communication (between you and your leaders, then between you and your team), whereas before it was more of a one-way street (between you and your manager].

You must be in frequent communication with your own 
manager and leadership in order to understand the bigger vision for the organization, and then you can drill down on what your team needs to accomplish and why. You must work closely with your staff to make sure the best possible work gets done by the best possible people, a large part of which is helping them to understand the big-picture impact of what they are doing.

You must look further into the future than you probably ever have before to see not just the project your team is working on today, but how it will connect to projects that will be done one to two years in the future.

In summary, these are the biggest transitions that occur when moving from IC to entry-level manager:

$\Rightarrow$ Let go of the immediatelquick sense of gratification that comes from doing/building/creating.

$\Rightarrow$ Accolades and recognition become less frequent as you move up.

$\Rightarrow$ You derive your sense of accomplishment from mentoring, growing, and furthering the work of your team and those around you.

$\Rightarrow$ Add value by removing roadblocks, streamlining processes, and helping others be productive.

$\Rightarrow$ Think one to two years out for your project and roadmap.

$\Rightarrow$ Help people connect their work to the parent organization or company, and help them see their individual impact and value.

ENTRY-LEVEL MANAGER TO MANAGER OF MANAGERS By the time you are promoted to become a manager of managers, you have some established management skills and experience under your belt. This means a less shocking 
transition than the one from IC to manager, but there are still plenty of changes to adapt to.

For example, you should be well practiced at letting go of micromanaging and instead trusting the people on your team to do good work [i.e., delegation]. As a manager of managers, however, the stakes increase on the work your direct reports are doing.

\section{Trusting your leaders}

Now, instead of your reports writing code that could be fixed in a day or two, they are making hiring decisions, managing performance, and driving strategy. A mistake here could have long-range and costly consequences, so you have to learn how to balance trusting your team with avoiding a disaster. If you override your leads, then it can erode trust in your team-and you quickly become a micromanager rather than a boss who empowers the team.

This ultimately comes down to knowing what calls really matter. Where do you need to be right, and where can you trust that your reports will make the right call or be able to course-correct from a bad call? If you override your direct reports, then you can erode their trust in you.

Knowing what calls matter comes from understanding what is most important, and how these decisions influence the overall strategy. To put things in perspective, zoom out two to three years into the future and ask yourself the following questions:

$\Rightarrow$ How do all of your teams fit together?

$\Rightarrow$ How should resources be distributed?

$\Rightarrow$ Which projects and people are critical to the success of 


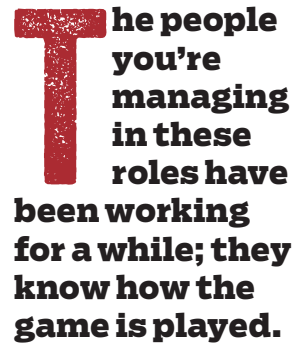

the organization's most important goals?

-What lessons do you need your managers to learn?

- Where can you allow them to take control and make mistakes?

$\Rightarrow$ What areas cannot fail and therefore need your oversight?

Once you have criteria around what matters, then the key is implementing the right checks and balances so you can feel confident in the decisions being made land have enough time to make adjustments when things go astray].

Your most important job becomes picking your leads. As the manager of one team, it's still possible to keep tabs on every single person. Not only do you know their names, but you probably also have at least a semi-clear idea of what they're spending their time on day to day.

As a manager of managers, you have not only your direct reports but also their teams under you. That is a lot to keep track of. In fact, it is more than you can keep track of.

This is why it becomes critically important to get the right people into a few key roles. In engineering teams, culture can be established at the manager/product level, but it can also come in the functional unit. As such, having a few really good senior people (technologists, project managers, product managers, UX leads, etc.) can help you maintain quality, excellence, and progress. Connect with those people and make sure they know how important they are.

The way you manage people will also change. The people you're managing in these roles have been working for a while; they know how the game is played. They are there for performance ratings, promotions, and 
compensation. As a result, you can be more transparent in your discussions with them.

That doesn't mean you can completely forget about coaching and mentoring, though. As a manager, it is still your job to help your direct reports achieve their career objectives.

\section{Planning for the future}

As you move up through the ranks at your organization, so do the people underneath you. If you are an entry-level manager who loses an engineer, that won't sink the ship. As the people who report to you take on increasingly important and hard-to-replace roles, however, you have to prepare for succession.

$\Rightarrow$ What will you do if your best [fill-in-the-blank] leaves?

-What can you do to help make your best people want to stay with your team?

$\Rightarrow$ Which resources do you need today, and what will you need a year from now?

$\Rightarrow$ Who is on your team right now who could move up in the future?

$\Rightarrow$ Which jobs don't exist today that you will need filled in the future?

$\Rightarrow$ Have any team members outgrown their roles, or have any of the roles changed enough that they are no longer filled by the right people?

You need to be continually looking into the future-not only in managing your people, but also in managing your team's work and objectives.

You need to know where you are going-again, you're looking at a two- to three-year time horizon-and then it's 
your job to set up systems that will allow you to get there. When you manage so many teams, you need to find ways to make it easy to know what is going on:

$\Rightarrow$ What metrics do you need to measure and pay attention to? Why?

$\Rightarrow$ How do you set up structures for visibility into progress?

This can be done in a variety of ways, from having skip-level meetings (those that involve managers and employees more than one level apart in the chain of command] to setting up reporting systems that automatically filter key data points up to you. Don't rely on just one method. Be creative, and make sure you are not getting a one-sided view.

Finally, how do you communicate those key metrics to your leadership? How will you communicate about success and failure?

Your success as a manager is now even harder to define, because what you do all day probably just looks like going to lots of meetings. There is almost no immediate, concrete output. It becomes even more critical that you get clarity from your leadership on what successful outcomes will look like for you and your team, and that you do the hard work today for big-picture results tomorrow.

Here is an overview of the transitions involved in becoming a manager of managers:

$\Rightarrow$ Continue learning to let go of control and allow people to make mistakes. Balance the importance of getting your way with the risk of undermining your people. Focus on the calls that really matter.

$\Rightarrow$ People management is still about mentoring, but there is a new level of transparency with your direct reports 
about the rewards of their work.

$\Rightarrow$ Your job is to think into the future. How do all of your teams fit together? How do changes in priorities affect the way people and resources are distributed? It is always better to be the person who can do more with less.

$\Rightarrow$ Succession planning comes into play. Make sure you have a solid plan to grow your leadership bench and maintain successors (or a plan) for all critical roles.

$\Rightarrow$ You are responsible for progress and execution. It's your job to make systems that work and track the right metrics and to share those results with your leadership.

\section{MANAGER OF MANAGERS TO ORGANIZATION LEADER}

When moving into a role that is several layers above the engineers, your role changes again. Now you are managing organizations consisting of unique teams that each may have their own culture, process, priorities, and mode of operation.

You have to decide where the teams should have similarities and what most benefits the organization as a whole. Each structure is different, so it helps to understand the principles that hold everything together: Is it about an aligned strategy, decision making, sharing resources, or something else?

Moving from manager to executive, is a huge shift in your career. Probably the biggest change you'll deal with is this: Your job is no longer the "what." Your job is now the "how."

This shift will define almost everything you do in this leadership role. When you manage hundreds of people, you become too far removed from the people doing the 
"what." Instead, the way you add value is by defining and streamlining the "how."

Your job is to make an entire organization successful. Here are some of the strategies that have worked well for me in practice.

\section{Establishing your team culture}

Give up now on the idea that you will be able to stay aware of everything going on within the teams you manage. If you try to make that part of your job, you will become the thing that holds back the talented people you have in placewhich is no way to retain top talent.

Instead, it is your job to establish a culture for your organization. Once you have the right people in the right places, then you need to step back and let them do the work.

What does it mean to establish a culture and values? Ask yourself these questions:

$\Rightarrow$ What does it mean to be in [team name]?

$\Rightarrow$ What do you stand for?

$\Rightarrow$ How should decisions be made?

$\Rightarrow$ How should issues be escalated?

$\Rightarrow$ What are the principles used to make tough calls?

Start by defining the values that you feel are most important to your organization. This will inform everything else. You want to start at the heart of the matter-what does it mean to be on this team and what do we stand forand that will inform the rest of your decisions.

For example, an Amazon manager told me he never does pre-reads for the meetings he runs. Instead, he sets aside the first few minutes of every meeting for all participants 
to read whatever document is being discussed in this meeting. In his view, very few people pre-read, and this ensures that the remaining meeting time is effective.

This might seem like a small detail, but it is indicative of someone who has a clear understanding of his own values and how he translates those values to his team. It is one small way that he executes his vision for his team culture.

You will find a large part of your role is creating and maintaining a structure for work to be done within and communicated about. Which meetings need to happen every week? Which happen monthly, quarterly, etc.? How often do you meet with the entire organization? How do you share the roadmap, and how often?

It is also critical to identify the culture-keepers in your organization. Are they the managers? Your senior engineers? Make sure you know who they are, but also that they know who they are. Show them that you understand their value. Help them see themselves as leaders and mentors.

Every decision you make at this level must be deliberate and reflect your organizational values. Once you make a decision, be open to reviewing it. If it's not working, iterate until it does.

Growth, mentoring, and people management How do you connect with your team when there are too many people to count?

The answer is, you try different things until you see what works best. And then you keep trying new things to avoid falling into a rut.

Here are a few ways to make yourself available to connect with the people who report to you: 


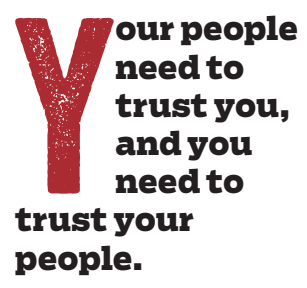

$\Rightarrow$ Office hours

$\Rightarrow$ Group lunches with different teams, randomly selected groups, etc.

$\Rightarrow$ Sporadic one-on-ones

$\Rightarrow$ Skip-level meetings

- Large group all-hands

$\Rightarrow$ Regular outbound communication via email, videos, etc.

You now manage more people than you could ever possibly keep track of in your head. You may work with teams distributed around the country or around the world. Does it matter if every single engineer working on a team within a team that you manage feels like he or she knows you? That might feel like an impossible challenge if you are managing hundreds of people. But don't forget, your role is to be the organization at this level. You represent the company and its goals.

Feeling a connection with you is a way for the people on your team to feel connected with the company they work for. No, you won't be friends or even on a first-name basis with everyone under you, but making an effort to be accessible will help people want to follow your lead over time. Based on my experience, the more people spend time with you and get to know you, the more effective you can be as a leader.

The name of the game now is trust. Your people need to trust you, and you need to trust your people.

Trust, but verify

But there is a caveat to that trust: You must also audit. Yes, you trust your managers, but you also do the work to check in. 
This can take a number of forms; for example, you might do a really deep dive into one or two programs on some regular cadence. You'll get a presentation from the key players and have time to ask questions about the work, timeline, and goals. You get the chance to review architecture, call out red flags, and confirm that progress is what is expected.

Just as startups have board meetings, you can also set up monthly updates or quarterly business reviews. My favorite format involves having highlights, lowlights, and sections for special topics.

These forms are a chance for you to answer questions for the people doing (or more likely, leading) the work, clarify your vision or goals for the project, and make sure your team is aligned. This is a much more sustainable way to work than just to assume you know what's going well and what's not, based on a bunch of status reports.

You cannot keep your eye on every single project and person every single day, but you can set up systems that allow you to keep in touch with the work being done on your team so that you can communicate effectively with your peers and your leadership.

If you work with a manager over time and don't feel that sense of trust, then it is critical to get that person out of that role or into another position as soon as possible. Your job just doesn't work without being able to trust your key leads.

Oversight and project reporting

Your managers are essentially acting as "you" land, in turn, the organization] when they speak to their teams. You need 
managers in place who are in line with your vision and who will accurately represent to their team what you tell them.

If there is dissonance between what you say and what the managers say, this will grow into a bad situation. Likewise, if what you say is different from what your peers or CEO say, that is also an opportunity to breed mistrust.

If there are managers who are not representing you accurately, then you need to correct them or replace them as soon as possible.

You should be upfront and clear with your managers about your expectations: What is their job? What is your job?

Their job is to deeply understand the company goals, how their team fits within those goals, and to communicate to you where things are working well and where they need help.

Your job is to support their decisions (which is possible when you know you have managers who innately understand the company goals] and to communicate clearly on behalf of the company's leadership.

With so many people and projects in your purview, it is important that you get regular status reports that are presented in the language of your organization [e.g., OKRs at Google, Red/Yellow/Green at Nordstrom, etc.]. This will enable you to share progress upward as efficiently as possible.

These reports, however, will give you only the simplest view of the work being done. To truly understand what is happening on your teams, you must maintain high-quality communication with the managers working for you.

One trick another executive told me was to select 
several projects to review (not exactly a deep dive, but devoting more time than just reading a report] every week. Look at key metrics, milestones, deadlines, and overall status. This is a good way to quickly and randomly audit the work being done, but it is an even better way to get insight into early red flags: Are there risks? Dependencies? Changes that need to be made?

Create a spreadsheet or other structured format for reviewing projects, so that each one can be judged and discussed using the same language.

\section{Talent review}

You are now managing high-level people in important roles. It is now even more important to think about the future of those people and those roles, and to have a plan should someone need to be replaced.

Make a point of identifying the high-potential people on your team. Who are they? Are they getting what they need? What will their path forward be? You can ask your managers for their recommendations, but it is likely that some standouts will be clear to you even from your relative distance.

You should also identify people who are criticalperhaps too critical-to the team's success. There should never be a single point of failure. If you would be in danger if a key person left, then you're in a really bad position that needs to be fixed right now. Find ways to create backups and have redundancy for your most critical people.

Make sure that you are involved in the hiring and evaluation process for your teams, even being part of interview loops for certain positions. Sit in and hear how 
lways look

for and check in with the smartest and most dissatisfied people on your team. your managers are giving feedback to employees and how managers are interviewing potential new hires. This could teach you a lot about your team and how the culture is being broadcast. It's an opportunity to catch big red flags that could have really long-term results.

When people do leave, always take the time to do exit interviews. Uncovering the flaws in your peoplemanagement system is just as important as finding the flaws in your programs and projects. There is always a push and a pull when someone leaves, so try to uncover both and identify a way to improve.

One friend told me always look for and check in with the smartest and most dissatisfied people on your team. They will alert you to problems within the team that might be small now but will be big problems for you in the future.

\section{Managing your own time and resources}

In this executive role, the work never stops. There will always be more than you can do. It is up to you to decide what is the most valuable use of your time and get out of the way of everything else. Hire people you trust who will help you be more effective by keeping the wheels turning while you focus on the most important tasks for yourself.

One important way you can do this is to make sure you have an exceptional assistant or other support staff. This should be someone who can read and respond to your email, because you will have a lot of it and most will not be worth your time. Get someone who is really invested in the job and who you can spend significant time with, allowing this person to understand how to make your life easier and more efficient. 
You could hire a chief of staff who acts as your number two and can speak on your behalf to make decisions for the team when you aren't available. This is a person who you'll spend a lot of time with, working through ideas and making sure you're always on the same page. This can be a formal or informal role, but it is essential to have someone you can count on in this role.

Talk to your CEO about the best uses of your time. Talk about this with your peers, too. How do they spend their time? What do they delegate?

Make sure that, even as you delegate many tasks, you continue to put a priority on time with your customers. With all the internal work you do, it can be easy to forget about the people outside of your organization who really matter. If you lose touch with your customers, you lose touch with your goals. You cannot lead your team effectively without knowing your customers, so it is worth your time to carve out regular parts of your schedule to get in tune with customers.

Working on a five-plus-year timeline When you think about concerns, you should be thinking further out. It is not uncommon to be focused on problems two to five years in the future. It can be a distraction to get mired in the details and tactical work, but when you can, you should delegate that to your very capable leads.

One way to help this process is simply to write it all down. Create a living document that defines your priorities and high-level goals. Write out your aspirational timeline and outline your strategy for achieving it. Update your document every year, and refer to it often. This will be your guide. 
Maintain an open communication loop from your team, to you, to your CEO, then back to you, and then your team. You are the in-between, and you will learn how to be successful by listening.

Make sure that you stay knowledgeable about your industry. You are no longer solving the problem in front of you; you are looking years into the future. Where is your industry going? What is your competition working toward? Is your company on track to fundraise, go public, multiply in size, completely change direction...?

Set goals that are continually growing. Make sure that every person on your team has goals. Some should be stretch goals, and some should be practical. Create a culture that places value on doing work that matters and gets the team's goals done, not on being the busiest or smartest or loudest person in the room.

Finally, create a roadmap and share it. You don't have to share every detail of what the next five years are going to look like, but share the vision of what you've been working on with your team and with your CEO. Get people on board with your vision and with you, so that you'll have their trust and enthusiasm on the journey.

This job isn't easy, but the rewards of executing amazing work on such a huge scale are some of the best you will ever experience.

Kate Matsudaira is an experienced technology leader. She worked in big companies such as Microsoft and Amazon and three successful startups (Decide acquired by eBay, Moz, and Delve Networks acquired by Limelight] before starting 
her own company, Popforms (https://popforms.com/], which was acquired by Safari Books. Having spent her early career as a software engineer, she is deeply technical and has done leading work on distributed systems, cloud computing, and mobile. She has experience managing entire product teams and research scientists and has built her own profitable business. She is a published author, keynote speaker, and has been honored with awards such as Seattle's Top 40 under 40. She sits on the board of acmqueue and maintains a personal blog at katemats.com.

Copyright $@ 2019$ held by ownerlauthor. Publication rights licensed to ACM. 